\title{
A Brief History of Natural Deduction
}

\author{
Francis JefFry Pelletier \\ Department of Philosophy, University of Alberta, Edmonton, Alberta, Canada T6G 2E5
}

Received July 1998 Revised February 1999

\begin{abstract}
Natural deduction is the type of logic most familiar to current philosophers, and indeed is all that many modern philosophers know about logic. Yet natural deduction is a fairly recent innovation in logic, dating from Gentzen and Jaśkowski in 1934. This article traces the development of natural deduction from the view that these founders embraced to the widespread acceptance of the method in the 1960s. I focus especially on the different choices made by writers of elementary textbooks - the standard conduits of the method to a generation of philosophers - with an eye to determining what the 'essential characteristics' of natural deduction are.
\end{abstract}

\section{Introduction}

In 1934 a most singular event occurred. Two papers were published on a topic that had (apparently) never before been written about, the authors had never been in contact with one another, and they had (apparently) no common intellectual background that would otherwise account for their mutual interest in this topic. ${ }^{1}$ These two papers formed the basis for a movement in logic which is by now the most common way of teaching elementary logic by far, and indeed is perhaps all that is known in any detail about logic by a number of philosophers (especially in North America). This manner of proceeding in logic is called 'natural deduction.' And in its own way the instigation of this style of logical proof is as important to the history of logic as the discovery of resolution by Robinson in 1965, or the discovery of the logistical method by Frege in 1879, or even the discovery of the syllogistic by Aristotle in the fourth century BC. ${ }^{2}$

Yet it is a story whose details are not known by those most affected: those 'ordinary' philosophers who are not logicians but who learned the standard amount of formal logic taught in North American undergraduate and graduate departments of philosophy. Most of these philosophers will have taken some (series of) logic courses that exhibited natural deduction, and may have heard that natural deduction is somehow opposed to various other styles of proof systems in some number of different ways. But they will not know why 'natural deduction' has come to designate the types of systems that are found in most current elementary logic textbooks, nor will they know why there are certain differences amongst the various textbooks and how these differences can nevertheless all be encompassed under the umbrella term 'natural deduction.'

The purpose of this article is to give a history of the development of this method of doing logic and to characterize what sort of thing is meant nowadays by the name.

1 Gerhard Gentzen (1934/5) and Stanasław Jaśkowski (1934).

2 Some scholars, e.g. Corcoran (1973), think that Aristotle's syllogism should be counted as a natural deduction system, on the grounds that there are no axioms but there are many rules. Although this might be a reasonable characterization of natural deduction systems, I wish to consider only those natural deduction systems that were developed in direct response to the 'logistical' systems of the late 1800 s and early 1900s. 
My view is that the current connotation of the term functions rather like a prototype: there is some exemplar that the term most clearly applies to and which manifests a number of characteristics. But there are other proof systems that differ from this prototypical natural deduction system and are nevertheless correctly characterized as being natural deduction. It is not clear to me just how many of the properties that the prototype exemplifies can be omitted and still have a system that is correctly characterized as a natural deduction system, and I will not try to give an answer. Instead I will focus on a number of features that are manifested to different degrees by the various natural deduction systems. My picture is that if a system ranks 'low' on one of these features, it can 'make up for it' by ranking high on different features. And it is somehow an overall rating of the total mount of conformity to the entire range of these different features that determines whether any specific logical system will be called a natural deduction system. Some of these features stem from the initial introduction of natural deduction in 1934; but even more strongly, in my opinion, is the effect that elementary textbooks from the 1950s had. There were of course some more technical works that brought the notion of natural deduction into the consciousness of the logical world of the 1950s and 1960s. But I will argue that the 'ordinary philosopher' of the time would have been little influenced by these works and that the huge sway that natural deduction holds over current philosophy is mostly due to the textbooks of the 1950s. The history of how these textbooks came to contain the material they do is itself an interesting matter, and I aim to detail this development of what is by now the most universally accepted method (within philosophy) of 'doing logic.'

\section{The concept of 'natural deduction'}

One meaning of 'natural deduction' focuses on the notion that systems employing it will retain the 'natural form' of first-order logic and will not restrict itself to any subset of the connectives nor any normal form representation. Although this is clearly a feature of the modern textbooks, we can easily see that such a definition is neither necessary nor sufficient for a logical system's being a natural deduction system. For, surely we can give natural deduction accounts for logics that have restricted sets of connectives, so it is not necessary. And we can have non-natural deduction systems (e.g. axiomatic systems) that contain all the usual connectives, so it is not sufficient.

Another feature of natural deduction systems, at least in the minds of some, is that they will have two rules for each connective: an introduction rule and an elimination rule. But again this can't be necessary, because there are many systems we happily call natural deduction which do not have rules organized in this manner. And even if we concocted an axiomatic system that did have rules of this nature, this would not make such a system become a natural deduction system. So it is not sufficient either.

A third feature in the minds of many is that the inference rules are 'natural' or 'pretheoretically accepted.' To show how widely accepted this feature is, here is what four elementary natural deduction textbooks across a forty year span have to say. Suppes (1957, viii) says: 'The system of inference... has been designed to correspond as closely as possible to the author's conception of the most natural techniques of informal proof.' Kalish and Montague $(1964,38)$ say that these systems 'are said to employ natural deduction and, as this designation indicates, are intended to reflect intuitive forms of reasoning.' Bonevac $(1987,89)$ says: 'we'll develop a system designed to simulate people's construction of arguments ... it is natural in the sense 
that it approaches... the way people argue.' And Chellas $(1997,134)$ says 'Because the rules of inference closely resemble patterns of reasoning found in natural language discourse, the deductive system is of a kind called natural deduction.' These authors are echoing Gentzen $(1934 / 35,74)$, one of the two inventors of natural deduction: 'We wish to set up a formalism that reflects as accurately as possible the actual logical reasoning involved in mathematical proofs.'

But this also is neither necessary nor sufficient. An axiom system with only modus ponens as a rule of inference obeys the restriction that all the rules of inference are 'natural', yet no one wants to call such a system 'natural deduction,' so it is not a sufficient condition. And we can invent rules of inference that we would happily call natural deduction even when they do not correspond to particularly normal modes of thought (such as is often done in modal logics, many-valued logics, relevant logics, and other non-standard logics).

As I have said, the notion of a rule of inference 'being natural' or 'pretheoretically accepted' is often connected with formal systems of natural deduction; but as I also said, the two notions are not synonymous or even co-extensive. This means that there is an interesting area of research open to those who wish to investigate what 'natural reasoning' is in ordinary, non-trained people. This sort of investigation is being carried out by a group of cognitive scientists, but their results are far from universally accepted (see Johnson-Laird and Byrne, 1991; Rips 1995).

There is also a history to the notion of 'natural deduction', and that history together with the way it was worked out by authors of elementary textbooks will account for our being drawn to mentioning such features of natural deduction systems and will yet also account for our belief that they are not definitory of the notion.

\section{Jaśkowski and Gentzen}

In his 1926 seminars, Jan Łukasiewicz raised the issue that mathematicians do not construct their proofs by means of an axiomatic theory (the systems of logic that had been developed at the time) but rather made use of other reasoning methods; especially they allow themselves to make 'arbitrary assumptions' and see where they lead. Łukasiewicz issued the challenge for logicians to develop a logical theory that embodied this insight but which yielded the same set of theorems as the axiomatic system than in existence. The challenge was taken up to Stanisław Jaśkowski, culminating in his (1934) paper. This paper develops a method-indeed, two methods - for making 'arbitrary assumptions' and keeping track of where they lead and for how long the assumptions are in effect. ${ }^{3}$ One method is graphical in nature, drawing boxes or rectangles around portions of a proof; the other method amounts to tracking the assumptions and their consequences by means of a book-keeping annotation alongside the sequence of formulas that constitutes a proof. In both methods the restrictions on completion of subproofs (as we now call them) are enforced by restrictions on how the boxes or book-keeping annotation s can be drawn. We would now say that Jaśkowski's system had two subproof-introduction methods: conditional-proof (conditional-introduction) and reductio ad absurdum (negationelimination). It also had rules for the direct manipulation of formulas (e.g. Modus Ponens). After formulating his set of rules. Jaśkowski remarks (p. 238) that the system

3 Some results of his had been presented as early as 1927, using the graphical method. 
' has the peculiarity of requiring no axioms' but that he can prove it equivalent to the established axiomatic systems of the time. (He shows this for various axiom systems of Łukasiewicz, Frege and Hilbert). he also remarks (p. 258) that his system is 'more suited to the purposes of formalizing practical [mathematical] proofs' than were the then-accepted system, which are 'so burdensome that [they are] avoided even by the authors of logical [axiomatic] systems.' Furthermore, 'in even more complicated theories the use of [the axiomatic method] would be completely unproductive.' Given all this, one could say that Jaśkowski was the inventor of natural deduction as a complete logical theory.

Working independently of Łukasiewicz and Jaśkowski, Gerhard Gentzen published an amazingly general and amazingly modern-sounding two-part paper in 1934/35. Gentzen's opening remarks are:

My starting point was this: The formalization of logical deduction, especially as it has been developed by Frege, Russell, and Hilbert, is rather far removed from the forms of deduction used in practice in mathematical proofs. Considerable formal advantages are achieved in return.

In contrast, I intended first to set up a formal system which comes as close as possible to actual reasoning. The result was a 'calculus of natural deduction' ('NJ' for intuitionist, ' $\mathrm{NK}$ ' for classical predicate logic)...

Like Jaśkowski, Gentzen sees the notion of making an assumption to be the leading idea of his natural deduction systems:

... the essential difference between $\mathrm{NJ}$-derivations and derivation s in the systems of Russell, Hilbert, and Heyting is the following: In the latter systems true formulae are derived from a sequence of 'basic logical formulae' by means of a few forms of inference. Natural deduction, however, does not, in general, start from basic logical propositions, but rather from assumptions to which logical deductions are applied. By means of a later inference the result is then again made independent of the assumption.

These two founding fathers of natural deduction were faced with the question of how this method of 'making an arbitrary assumption and seeing where it leads' could be represented. As remarked above, Jaśkowski gave two methods. Gentzen also contributed a method, and there is one newer method. All of the representational methods used in today's natural deduction system are variants on one of these four.

To see the four representations in use let's look at a simple propositional theorem: $(((\mathrm{P} \supset \mathrm{Q}) \&(\sim \mathrm{R} \supset \sim \mathrm{Q})) \supset(\mathrm{P} \supset \mathrm{R})) .{ }^{4}$ Since the main connective is a conditional, the most likely strategy will be to prove it by a rule of conditional introduction. But to apply this rule one must have a subproof that assumes the conditional's antecedent and ends with the conditional's consequent. All the methods will follow this strategy; the differences among them concern only how to represent the strategy. In Jaśkowski's graphical method, each time an assumption is made it starts a new portion of the proof

4 Jaśkowski's language contained only conditional, negation, and universal quantifier, so the use of \& here is a certain liberty. But it is clear what his method would do if \& were a primitive. I call the rule of \&-elimination 'simplification'. 
which is to be enclosed with a rectangle (a 'subproof'). The first line of this subproof is the assumption... in the case of trying to apply conditional introduction, the assumption will be the antecedent of the conditional to be proved and the remainder of this subproof will be an attempt to generate the consequent of that conditional. If this can be done, then Jaśkowski's rule conditionalization says that the conditional can be asserted as proved in the subproof level of the box that surrounds the one just completed. So the present proof will assume the antecedent, $((\mathrm{P} \supset \mathrm{Q}) \&(\sim \mathrm{R} \supset \sim \mathrm{Q}))$, thereby starting a subproof trying to generate the consequent, $(P \supset R)$. But this consequent itself has a conditional as main connective, and so it too should be proved by conditionalization with a yet-further-embedded subproof that assumes its antecedent, $\mathrm{P}$, and tries to generate its consequent, R. As it turns out, this subproof calls for a yet further embedded subproof using Jaśkowski's reductio ad absurdum.

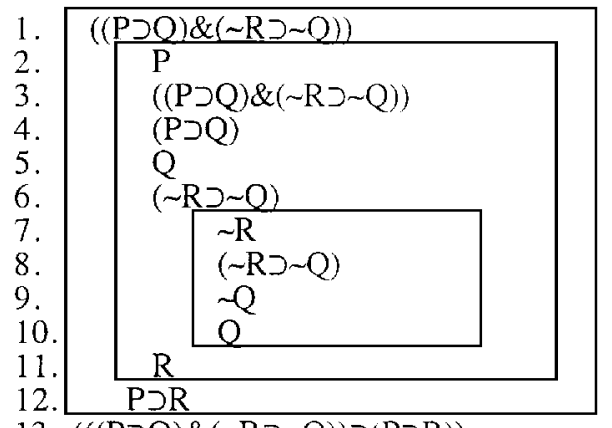

13. $(((\mathrm{P} \supset \mathrm{Q}) \&(\sim \mathrm{R} \supset \sim \mathrm{Q})) \supset(\mathrm{P} \supset \mathrm{R}))$
Supposition

Supposition

1, Repeat

3, Simplification

2,4 Modus Ponens

3, Simplification

Supposition

6, Repeat

7,8 Modus Ponens

5, Repeat

7-10 Reductio ad Absurdum

2-11 Conditionalization

1-12 Conditionalization

To make the ebb and flow of assumptions coming into play and then being 'discharged' work, one needs restrictions on what formulas are available for use with the various rules of inference. Using the graphical method, Jaśkowski mandated that any 'ordinary rule' (e.g. Modus Ponens) is to have all the formulas required for the rule's applicability be in the same rectangle. If the relevant formulas are not in the right scope level, Jaśkowski has a rule that allows lines to be repeated from one scope level into the next most embedded rectangle, but no such repetitions are allowed using any other configuration of the rectangles. The 'non-ordinary' rules of Conditionalization and Reductio require that the subproof that is used to justify the rule's applicability be immediately embedded one level deeper than the proposed place to use the rule. There are also restrictions that make each rectangle, once started, be completed before any other, more inclusive, rectangles can be completed. We need not go into these details here. A formula is proved only 'under certain suppositions' unless it is outside of any boxes, in which case it is a theorem - as the above demonstration proves about line \#13.

This graphical method was streamlined somewhat by Fitch (1952), as we will see in more detail below, and proofs done in this manner are now usually called 'Fitch diagrams.' (Fitch does not have the whole rectangle, only the left vertical line; and he draws a line under the first formulae of a subproof to indicate explicitly that it is an assumption for that subproof.) This method, with some slight variations, was then followed by Copi (1954), Anderson and Johnstone (1962), Kalish and Montague (1964), Thomason (1970), Leblanc and Wisdom (1972), Kozy (1974), Tapscott (1976), Bergmann et al. (1980), Klenk (1983), Bonevac (1987), Kearns (1988), Wilson (1992), and many others. 
Jaśkowski's second method (which he had hit upon somewhat later than the graphical method) was to make a numerical annotation on the left-side of the formulas in a proof. This is best seen by example; and so we will re-present the previous proof. but a few things were changed by the time Jaśkowski described this method. First, he changed the statements of various of the rules and he gave them new names: Rule I is now the name for making a supposition, Rule II is the name for conditionalization, Rule III is the name for modus ponens, and Rule IV is the name for reductio ad absurdum. (Rules V, VI, and VII have to do with quantifier elimination and introduction). ${ }^{5}$ Some of the details of these changes to the rules are such that it is no longer required that all the preconditions for the applicability of a rule of inference must be in the same 'scope level' (in the new method this means being in the same depth of numerical annotation), and hence there is no longer any requirement for a rule of repetition. To indicate that a formula is a supposition, Jaśkowski now prefixes it with ' $S$ '.

$\begin{array}{lll}1.1 . & S((\mathrm{P} \supset \mathrm{Q}) \&(\sim \mathrm{R} \supset \sim \mathrm{Q})) & \text { I } \\ 2.1 . & (\mathrm{P} \supset \mathrm{Q}) & \& \mathrm{E} 1 \\ 3.1 . & (\sim \mathrm{R} \supset \sim \mathrm{Q}) & \text { \&E } 1 \\ 4.1 .1 . & S \mathrm{P} & \text { I } \\ 5.1 .1 . & \mathrm{Q} & \text { III } 4,2 \\ 6.1 .1 .1 . & \mathrm{S} \sim \mathrm{R} & \text { I } \\ 7.1 .1 .1 . & \sim \mathrm{Q} & \text { III } 6,3 \\ 8.1 .1 . & \mathrm{R} & \text { IV } 5,7,6 \\ 9.1 . & \mathrm{P} \supset \mathrm{R} & \text { II } 4,8 \\ 10 . & (((\mathrm{P} \supset \mathrm{Q}) \&(\sim \mathrm{R} \supset \sim \mathrm{Q})) \supset(\mathrm{P} \supset \mathrm{R})) & \text { II } 1,9\end{array}$

It can be seen that this second method is very closely related to the method of rectangles. (And much easier to typeset!) Its only real drawback concerns whether we can distinguish different subproofs which are at the same level of embedding. A confusion can arise when one subproof is completed and then another started, both at the same level of embedding. In the graphical method there will be a closing of one rectangle and the beginning of another, but here it could get confused. Jaśkowski's solution is to mark the second such subproof as having ' 2 ' as its rightmost numerical prefix. This makes numerals be superior to using other symbols in this role, such as an asterisk. As we will see in section 5, this representational method was adopted by Quine (1950a), who used asterisks rather than numerals thus leading to the shortcoming just noted.

One reason that this book-keeping method of Jaśkowski did not become more common is that Suppes (1957) introduced a method (which could be seen as a variant on the method, but which I think is different enough in both its appearance and in its metalogical properties that it should be called a distinct method) using the line numbers of the assumption s which any given line in the proof depended upon, rather than asterisks or arbitrary numerals. In this third method, when an assumption is made its line number is put in set braces to the left of the line (its 'dependency set'). The application of 'ordinary rules' such as \& E and Modus Ponens make the resulting formula inherit the union of the dependencies of the lines to which they are applied, whereas the 'scope changing' rules like $\supset$ I and Reductio delete the relevant assumption's line number from the dependencies. In this way, the 'scope' of an

5 For purposes of the example we continue attributing a rule of \&-elimination to Jaśkowski, even though he did not have $\&$ in his system. 
assumption is not the continuous sequence of lines that occurs until the assumption is discharged by a $\supset$ I or $\sim$ I rule, but rather consists of just those (possibly noncontiguous) lines that 'depend upon' the assumption. Without using Suppes's specific rules, we can get the flavour of this style of representation by presenting the above theorem as proved in a Suppes-like manner.

$\begin{array}{llll}\{1\} & 1 . & ((\mathrm{P} \supset \mathrm{Q}) \&(\sim \mathrm{R} \supset \sim \mathrm{Q})) & \\ \{1\} & 2 . & (\mathrm{P} \supset \mathrm{Q}) & \& \mathrm{E} 1 \\ \{1\} & 3 . & (\sim \mathrm{R} \supset \sim \mathrm{Q}) & \& \mathrm{E} 1 \\ \{4\} & 4 . & \mathrm{P} & \\ \{1,4\} & 5 . & \mathrm{Q} & \supset \mathrm{E} 4,2 \\ \{6\} & 6 . & \sim \mathrm{R} & \\ \{1,6\} & 7 . & \sim \mathrm{Q} & \supset \mathrm{E} 6,3 \\ \{1,4\} & 8 . & \mathrm{R} & \text { Reductio } 5,7,6 \\ \{1\} & 9 . & \mathrm{P} \supset \mathrm{R} & \supset \mathrm{I} 4,8 \\ \emptyset & 10 . & (((\mathrm{P} \supset \mathrm{Q}) \&(\sim \mathrm{R} \supset \sim \mathrm{Q})) \supset(\mathrm{P} \supset \mathrm{R})) & \supset \mathrm{I} 1,9\end{array}$

The appearance of the empty set as the dependency set for line 10 shows that it is a theorem. This method seems much superior to the other book-keeping method, and must have seemed so to other writers since they adopted this method rather than the Jaśkowski way. Some version of Suppes's style of proof was adopted by Lemmon (1965), Mates (1965), Pollock (1969), Myro et al. (1987), Bohnert (1977), Chellas (1997) and many others.

The fourth method was presented by Gentzen. Proofs in the $\mathrm{N}$ calculi (the natural deduction calculi) are given in a tree format with sets of formulas appearing as nodes of the tree. The root of the tree is the formula to be proved, and the 'suppositions' are at the leafs of the tree. The following is a tree corresponding to the example we have been looking at, although it should be mentioned that Gentzen's rule for indirect proofs first generated $\perp$ ('the absurd proposition') from the two parts of a contradiction, and then generated the negation of the relevant assumption.

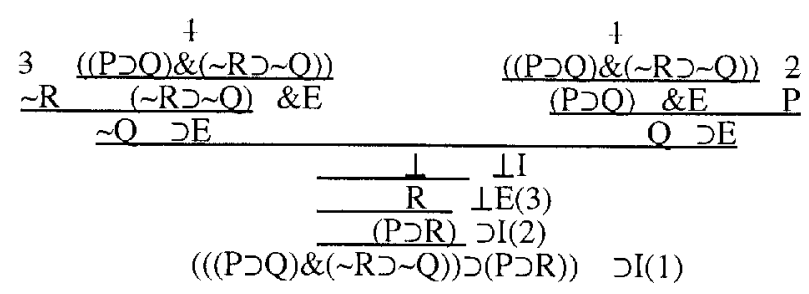

The lines indicate a transition from the upper formula(s) to the one just beneath the line, using the rule of inference indicated to the right of the lower formula. (We might replace these horizontal lines with vertical or splitting lines to more clearly indicate tree-branches, and label these branches with rule of inference responsible, and the result would look even more tree-like). Gentzen uses the numerals on the leafs as a way to keep track of subproofs. Here the main antecedent of the conditional to be proved is entered (twice, since there are two separate things to do with it) with the numeral ' 1 ', the antecedent of the consequent of the main theorem is entered with numeral ' 2 ', and the formula $\sim \mathrm{R}$ (to be used in the reductio part of the proof) is entered with numeral ' 3 '. When the relevant rule 'scope changing' is applied (indicated by citing the numeral of that branch as part of the citation of the rule of inference, in parentheses) this numeral gets 'crossed out', indicating that this subproof is finished. 
As Gentzen remarks, very complex proofs show that 'the calculus $\mathrm{N}$ lacks a certain formal elegance' because of the book-keeping matters. However, he says, this has to be put against the following advantage s of $\mathrm{N}$ systems (p. 80):

(1) A close affinity to actual reasoning, which had been our fundamental aim in setting up the calculus.

(2) In most cases the derivations for true formula are shorter in our calculus than their counterparts in the logistic calculi. This is so primarily because in logistic derivations one and the same formula usually occurs a number of times (as part of other formulae), whereas this happens only very rarely in the case of $\mathrm{N}$-derivations.

(3) The designations given to the various inference figures [rules of inference] make it plain that our calculus is remarkably systematic. To every logical symbol belongs precisely one inference figure which 'introduces' the symbol - as the terminal symbol [main connective] of a formula - and one which 'eliminates' it.

The Gentzen tree method did not get used much in elementary logic books, with the exception of Curry, 1963 (who said his book was for 'graduate students of philosophy') and van Dalen, 1980 (which is for advanced undergraduates in mathematics). But the method enjoyed some play in the more technical works on natural deduction, especially Prawitz (1965) and the many works of Curry. It is also used when comparisons are made to other styles of proving in various of the Russian works (e.g. Maslov (1969) and Mints (1997)). But even in works expanding on Gentzen, it is far more common to use his sequent calculus than his natural deduction systems. (See section 11). In any case, this method of representing natural deduction proofs is not at all common any more.

Gentzen's was the first use of the term 'natural deduction' to describe logical systems, and therefore it is only natural that his characterization would strongly influence what is to be given the title in any future use of the term. But it is not correct to say, for instance, that all natural deduction systems must contain precisely the specific rules that Gentzen proposed, for we know that there are many different ways to posit the rules of inference that lead to the same effect. Nor is it even correct to say that a natural deduction system cannot contain axioms. In fact, Gentzen's own formulation of $\mathrm{NK}$, the natural deduction system for classical logic, was obtained by taking the intuitionistic NJ system and adding all instances of $(\Psi \vee \neg \Psi)$ as 'basic formulae' (axioms). ${ }^{6}$ He remarks that he could have equivalently added doublenegation elimination as a further rule, but that such an elimination of two negations at once violated his notion of admissibility of rules. (Modern treatments of natural deduction do not normally have these scruples about admissibility of rules).

In this same article, Gentzen also introduced another type of proof system: the sequent calculus. This is a topic to which we shall return in section 11 .

\section{Nine choice points in natural deduction systems}

In the next sections we will see how the Jaśkowski/Gentzen goal of employing natural deduction proof systems rather than 'logistical' (axiomatic) systems came to

6 And so I would say that the characterization of 'natural deduction' as being directly opposed to 'having axioms' (e.g. by Corcoran 1973, 192) cannot be quite correct. 
pass into the teaching of logic to generation s of (mostly North American ${ }^{7}$ ) philosophy and mathematics students. But first, with an eye to bringing some order to the bewilderingly diverse array of details in the different systems, in this sub-section I will lay out some 'choice points' that are differently followed by our different authors. It is perhaps because there is such an array of differing options chosen by authors within the general natural deduction framework that it is so difficult to give an unambiguously straightforward set of necessary and sufficient conditions for a proof system's being correctly called a natural deduction system.

Before recounting the various choices available to a developer of natural deduction systems, let me impose a matter of terminology. I will use the introduction and elimination rule-names ( $\supset \mathrm{I}, \& \mathrm{E}, \forall \mathrm{I}$ and so on) in the way that Gentzen uses them, with the exception of the negation rules. Without deviating too much from Gentzen, but being more in line with the later developments, we will use the negation introduction and elimination rules as follows:

From $\sim \sim \phi$ infer $\phi(\sim \mathrm{E})$

From an immediately embedded subproof $[\phi \ldots \Psi \ldots \sim \Psi]$, infer $\sim \phi(\sim \mathrm{I})$

(The last rule, requiring the demonstration that an assumption of $\phi$ leads to a contradiction, will recognized as a negation-introducing version of Reductio ad Absurdum, while the first is Double Negation elimination). Some of our writers use different names for the same rules, as for example Modus Ponens (MP) for $\supset$ E; while others call somewhat different rules by Gentzen's names. And there are a number of other rules that have been proposed ${ }^{8}$ One particularly important difference concerns the quantifier rules, especially the elimination of the existential quantifier. I will discuss this soon, as one of the major choice points. In this discussion I will distinguish a rule of Existential Instantiation (EI) from Gentzen's Existential Elimination $(\exists \mathrm{E})$.

All the systems we will consider below have a rule of $\supset$ I, which introduces a conditional formula if one has an immediately-embedded subproof that assumes the antecedent of the conditional and ends with the consequent of the conditional. ${ }^{9}$ This means that all these systems have a mechanism by means of which an assumption can be made, and they all have some means of keeping track of the 'scope' of an assumption (that is, a way of demarcating a subproof from the one that encompasses it, and to demarcate two separate and independent subproofs both of which are encompassed by the same subproof). This much is common to all, although the rule I might be called CP ('conditional proof') or Cd ('conditional derivation'), etc. and although the ways of demarcating distinct subproofs may differ. We have already seen, from Jaśkowski, Suppes, and Gentzen, four basic methods of representing proofs and

7 At the time, British philosophy schools, and those heavily influenced by them, tended instead to study 'philosophy of logic' as presented by Strawson (1952). Those who studied logic on the Continent during this period mostly worked in the axiomatic framework.

8 Gentzen did not have a $\equiv$ (material equivalence) in his language. Many of the more recent authors do, and therefore have rules governing its use, but we will not remark on this further.

9 But as I remarked above in section 1, we could have a natural deduction system without a conditional and hence with no rule $\supset$ I. For example, we could have 'nand' $(\Phi \uparrow \Psi)$ as the only connective. An appropriate rule of $\uparrow I$ might be: if from the assumption of $\Phi$ one can derive $(\Psi \uparrow \Psi)$, then in the next outer scope we can conclude $(\Phi \uparrow \Psi)$ by $\uparrow \mathrm{I}$ [and a symmetrical form that assumes $\Psi$, derive $(\Phi \uparrow \Phi)$ and concludes $(\Phi \uparrow \Phi)]$. A rule of $\uparrow E$ might be: from $(\Phi \uparrow \Psi)$ and $\Phi$, infer $(\Psi \uparrow \Psi)$ [and a symmetrical form that eliminates from the other side]. And probably a sort of reductio rule will be desired: if from the assumption of $(\Phi \uparrow \Phi)$ we can infer both $\Psi$ and $(\Psi \uparrow \Psi)$, then on the next outer scope we can infer $\Phi$. It can be seen that the $\uparrow \mathrm{I}$ and reductio rules are of the natural deduction sort because they involve the construction of a subproof and the subproof involves making an assumption. See also Price (1962) for natural deduction rules for Sheffer strokes and a discussion of the issues involved in constructing them. 
hence the four basic ways to keep track of subproofs. Which of these methods to adopt is what I refer to as the first choice point for natural deduction system-builders.

The second choice point concerns whether to allow axioms in addition to the rules. Despite the fact that Jaśkowski found 'no need' for axioms, Gentzen did have them in his NK. And many of the authors of more modern textbook sendorse methods that are difficult to distinguish from having axioms. For example, as a primitive rule many authors have a set of 'tautologies' that can be entered into a proof anywhere. This is surely the same as having axioms. Other authors have such a set of tautological implications together with a rule that allows a line in a proof to be replaced by a formula which it implies according to a member of this set of implications. And it is but a short step from here to add to the primitive formulation of the system a set of 'equivalences' that can be substituted for a subpart of an existing line. A highly generalized form of this method is adopted by Quine (1950a), where he has a rule TF ('truth functional inference') that allows one to infer 'any schema which is truthfunctionally implied by the given line(s) ${ }^{10} \cdot{ }^{11}$ Although one can detect certain differences amongst all these variants I have just mentioned here, I would classify them all as being on the 'adopt axioms' side of this second dimension. Of course, from a somewhat different point of view one might separate Quine from the other 'axiomatic' systems in that he does not have any list of tautological implications to employ, and instead formulates this as a rule. We might note that, in the propositionallogic, Quine in fact has no real need for the rule of conditionalization. For, everything can be proved by the rule TF. (Any propositional theorem follows from the null set of formulas by TF).

Related to the choice of allowing axioms is a third choice of how closely the system is to embrace the int-elim ideal of Gentzen: that there be an introduction rule and an elimination rule for each connective, and that there be no other rules. ${ }^{12}$ There are complete sets of int-elim rules, so we know that the class of all valid inferences can be generated out of a set of primitive int-elim inferences. But there are other sets of primitive rules that do not obey the int-elim ideal but also can generate the set of all valid inferences. (Without going to Quine's extreme of just allowing all propositional inferences to be primitively valid). As we will see, most textbook writers do not follow the int-elim ideal, but instead have a large number of 'overlapping' rules (presumably for pedagogical reasons). And so the third choice is a matter of deciding how far to deviate from having all rules be int-elim.

A fourth choice point in the propositionallogic is to determine which propositional rules shall require a subproof as a precondition. Although we've seen that all systems have a $\supset$ I rule, and that this requires a subproof as a precondition, there is considerable difference on the other rules ... even in those systems that, unlike Quine's, actually have a set of propositional rules. For example, Gentzen's rule of $\vee \mathrm{E}$ is:

\section{From $\phi \vee \Psi$ and embedded subproofs $[\phi \ldots \theta]$ and $[\Psi \ldots \theta]$ infer $\theta$}

10 Quine's TF rule allows one to infer anything that follows from the conjunction of lines already in the proof.

11 In his (1950b, fn. 3) Quine says that the most important difference between him and Gentzen is in the formulation of the existential quantifier elimination rule, and that the difference between Quine's TF and Gentzen's introduction and elimination rules for all the connectives 'is a trivial matter.' It is not clear to me that Gentzen would agree with this, for he heavily emphasized the int-elim ideal as a crucial feature of natural deduction. Cellucci (1995, 315-316) agrees with me in this evaluation.

12 As remarked above, Gentzen did not think this could be done in an appropriate manner for classical logic. In his mind this showed that classical logic was not 'pure' in the same way that intuitionisticlogic was. 
which requires subproofs. But it is clear that we could do equally well with 'separation of cases':

\section{From $\phi \vee \Psi$ and $\phi \supset \theta$ and $\Psi \supset \theta$, infer $\theta$}

where there are no required subproofs. (In the presence of $\supset$ I the two rules are equivalent). Gentzen's natural deduction had required subproofs for $\supset \mathrm{I}, \vee \mathrm{E}, \exists \mathrm{E}$, and his version of negation introduction. It is possible to have a natural deduction system with $\supset$ I as the only subproof-requiring rule of inference: Quine's (1950a) is like that. But on the opposite hand, some authors have not only the four subproofrequiring rules of Gentzen (with the $\sim$ I rule introduced at the beginning of this subsection replacing Gentzen's), but in addition have subproof-requirin g rules for $\forall$ I, $\equiv \mathrm{I}$, and for a version of Reductio that eliminates negations. And pretty much any intermediate combination of the two types of rules can be found in some author or other.

There are a number of choice points concerning the use of variables in first-order natural deduction systems. But before we come to these choices, a few words of background are in order. The proper treatment of variables in natural deduction proof systems is much more involved than in some other proof systems. For example, even though semantic tableaux systems retain the 'natural form' of formulas just as much as natural deduction systems do, because tableaux systems are decompositional in nature and so use only elimination rules, they need not worry about $\forall \mathrm{I}$ and its interaction with $\exists \mathrm{E}$ or free variables in assumptions and premises. This means in particular that no tableaux proof will ever try to infer a universally quantified formula from any instance of that formula ... only a quantifier introduction rule would try to do that. Hence, tableaux systems need not worry about the proper restrictions on variables that would allow such an inference. But natural deduction systems do allow this; indeed, it is one of the features of natural deduction theorem proving that it can construct direct proofs of conclusions, rather than trying to show unsatisfiability (as tableaux and resolution proofs do).

The treatment of variables in natural deduction is also more involved than in resolution systems. Resolution converts formulas to a normal form which eliminates existential quantifiers in favour of Skolem functions. But because the Skolem functions explicitly mention all the universal quantifiers that had the original existential quantifier in their scope, this information will be present whenever a formula is used in an inference step. And the unification-of-variables rule will preserve this information as it generates a formula with the most general unifier. But in a natural deduction system this information is only available by relative placement of quantifiers. And these quantifiers could become separated from each other when rules of inference are used on them. Thus the formula $\forall \mathrm{x}(\mathrm{Fx} \supset \exists \mathrm{yGy})$ might have $\forall \mathrm{E}$ applied to it to yield $\mathrm{Fa} \supset \exists \mathrm{yGy}$, and Fa might be somewhere in the proof so that $\supset \mathrm{E}$ could be used to yield $\exists$ yGy. But now this resulting formula has no indication that it is actually somehow dependent on the choice of $x$ in the first formula (namely ' $a$ ', from the other formula). In a resolution-style proof the first formula would represented as $\sim \mathrm{F}(\mathrm{x}) \vee \mathrm{G}(\operatorname{sk}(\mathrm{x})$ ) (with implicit universal quantification), and when doing a resolution with Fa, the result would be $\mathrm{G}(\operatorname{sk}(\mathrm{a}))$, since $a$ would be the most general unifier with $x$, and this resulting formula explicitly mentions the instances of the universally quantified variable which is logically responsible for this formula.

But none of this 'Skolem information' is available in a natural deduction proof. 
Formulas are simplified by using the elimination rules; and formulas are made more complex by using the introduction rules. All along, variables and quantifiers are added or deleted, and no record is kept of what variable used to be in the scope of what other universally quantified variable. This all shows that the proper statement of a universal quantifier introduction rule, $\forall \mathrm{I}$, is quite complex; and it interacts with the way an existential quantifier elimination rule, $\exists \mathrm{E}$, is stated. It furthermore is affected by whether one allows a new category of terms into the language, just for helping in this regard. (Those who take this route call these new terms 'arbitrary names' or 'parameters').

We are now ready to resume our discussion of choice points in developing natural deduction systems. A fifth choice point involves whether to have more than one quantifier. Jaśkowski only had one quantifier, $\forall$, and therefore did not need to worry about its interaction with $\exists$. This route is not taken much in the elementary logic teaching literature, although Mates (1965) did have only $\forall$ in his primitive vocabulary (therefore he had only $\forall \mathrm{I}$ and $\forall \mathrm{E}$ as primitive rules). But he soon introduced the defined existential quantifiers and derived rules for introducing and eliminating them.

A sixth choice point concerns whether to use subordinate proofs as the precondition for $\exists \mathrm{E}$. We've seen that in the propositional case, there appears to be no 'logical' issue involved in whether to use $\vee \mathrm{E}$ or use separation of cases... merely (perhaps) some aesthetic issue. And much the same can be said about the other propositional rules for which some writers require a subproof (so long as $\supset$ I is present). But in the case of quantifiers there is a logical difference. Gentzen's rule for Existential Quantifier Elimination $(\exists \mathrm{E})$ is :

\section{( $\exists$ E) From $\exists x \phi x$ and a subproof $[\phi \alpha \ldots \Psi]$, infer $\Psi$}

(with certain restrictions on $\alpha$ and on the variables occurring in $\Psi$ ). That is, to eliminate an existentially quantified formula, we assume an 'arbitrary' instance of it in a subproof. Things that follow from this arbitrary instance (and which obey the restrictions on the variables) can be 'exported' out to the subproof level in which the existentially quantified formula occurred. But an alternative way to eliminate an existential quantifier could be by Existential Instantiation (EI):

\section{(EI) From $\exists x \phi x$, infer $\phi \alpha$}

(with certain restrictions on $\alpha$ ). Here the instance is in the same subproof level as the existentially quantified formula. This in turn has various logical implications. For instance, proofs in a system employing this latter rule do not obey the principle that each line of a proof is a semantic consequence of all the assumption s that are 'active' at that point in the proof. For, even if $\exists \mathrm{xFx}$ were semantically implied by whatever active assumptions there are, it is not true that Fy will be implied by those same assumptions, since the rule's restriction on variables requires that y be new. But in systems that have a $(\exists \mathrm{E})$-style rule, the situation is different. For, the 'arbitrary instance' becomes yet another active assumption of all the formulas in that subproof, and the restrictions on the variables that can occur in $\Psi$ when it is 'exported' ensure that this formula does not semantically depend upon the arbitrary instance. Quine's system used the (EI)-style of rule-he called it Existential Instantiation (EI) - and systems that have such a rule are now usually called 'Quine-systems'. Systems using Gentzen's rule could be called 'Gentzen-systems', but when referring especially to this 
particular matter, they are more usually called 'Fitch-systems' (see below). In a Quine system, without further restrictions, it is possible to prove such formulas as ( $\exists \mathrm{xFx} \supset \mathrm{Fy}$ ) by assuming the antecedent, applying EI to this formula, and then using $\supset$ I. Yet under most interpretations of this formula, it is not semantically valid. It is rather more difficult to give adequate restrictions for Quine systems than for Fitch systems, as can be intuitively seen from the following quotation from Quine (1950a, 164), when he is discussing just this sort of inference:

Clearly, then, UG and EI need still further restriction, to exclude cases of the above sort in which a flagged variable survives in premiss or conclusion to corrupt the implication. A restriction adequate to this purpose consists merely in regarding such deductions as unfinished. The following is adopted as the criterion of a finished deduction: No variable flagged in the course of a finished deduction is free in the last line nor in any premiss of the last line.

Even before investigating Quine's notion of flagging variables we can see that there is a tone of desperation to this. And in fact the method specified in the first edition of Quine (1950a) was changed in the second edition of $1959 .{ }^{13}$

A seventh choice point is whether to have a subproof introduction rule for $\forall$ I. The idea behind a rule of $\forall \mathrm{I}$ is that one should derive a formula with an 'arbitrary variable' in order to conclude that the formula is true for everything. At issue is the best way to ensure that the variable is 'arbitrary'. One way would be to stay within the same subproof level, requiring that the 'arbitrary variable' must pass such tests as not occurring free in any premise or undischarged assumption. Yet another way, however, would be to require a subproof with a special marking of what the 'arbitrary variable' is going to be and then require that no formula with that variable free can be reiterated into, or used in, that subproof, and that no existential instance of a formula that uses that variable can be assumed in an embedded subproof, as when one does this with the intention of being able to apply $\exists \mathrm{E}$, within this subproof. (This last discussion - of having embedded subproofs to apply $\exists \mathrm{E}$ inside the suggested version of $\forall \mathrm{I}$ that requires a subproof-shows that there is an interaction between whether one has a subproof-introducing $\forall$ I rule and also a subproof-introducin $g \exists \mathrm{E}$ rule. As one can see, there are four possibilities here, and each of them is taken by some elementary textbook author over the last 50 years.)

An eighth choice point concerns whether to require premises and conclusion (of the whole argument) to be sentences (formulas with no free variables), or whether they are allowed to contain free variables. (If the requirement were imposed, then the fact that the semantically invalid $(\exists x F x \supset$ Fy) can be proved in a Quine system might not be a problem, since it couldn't be a conclusion of a 'real' argument because it had a free

13 The preface to the Third Edition (1972) of this book says (p. vi): 'The second edition, much revised, should have come out in 1956, but was delayed three years by an inadvertent reprinting of the old version.' Had it come out in 1956, closer to the publication of Copi's (1954) with its logically incorrect combination of EI and UG, perhaps more of the budding early logicians would have understood the various correct ways to present the restrictions. As it was, the combination of the bizarre and baroque method of the first edition of Quine's (1950a), the logically incorrect method in Copi (1954), and the radically different-looking method of Fitch (1952) - the Gentzen subproof method - made for a rather mysterious stew of ideas. Anellis (1991) contains a discussion of the different versions of EI, $\exists$ E, and $\forall$ I that were in the air during the 1950 s and 1960 s. Despite the fact that there are many places where Anellis and I differ on interpretation of facts, and indeed even on what the facts are, nonetheless I believe that his article is a fascinating glimpse into the sociology of this period of logic pedagogy. 
variable.) If they are allowed to contain free variables, then one needs to decide how sentences containing them are to be semantically interpreted (e.g. whether they are interpreted as if they were universally quantified or as if existentially quantified.) Such a decision will certainly dictate the statement of quantifier rules and particularly of the restrictions on variables.

Yet a ninth choice point is related to the just-mentioned one. This is whether to have an orthographically distinct category of 'arbitrary names' or 'parameters' to use with the quantifier rules. For example, using a Quine-system we might say that the EI rule must generate an instance using one of these arbitrary names, rather than an ordinary variable or ordinary constant. Or, instead of this one might require that using $\forall$ I to infer $\forall \mathrm{xFx}$ requires having $\mathrm{F} \alpha$, where $\alpha$ is one of these arbitrary names. It can here be seen that the notion of 'arbitrary name' encompasses two quite distinct, and maybe incompatible, ideas. One is relevant to existential quantifiers and means 'some specific object, but I don't know which', while the other is relevant to universal quantifiers and means 'any object, it doesn't matter which.' These choices interact with the eighth choice point on the issue of free variables in premises and conclusions, and with the background issue in the sixth choice point - the status of the metalogical claim that each line should be a valid semantic entailment of all the assumptions upon which it depends. Here's what Suppes $(1957,94)$ says about the issue.

If we interpret ambiguous names in the same way that we interpret proper names and free variables, then not every line of a derivation is a logical consequence of the conjunction of the premises on which it depends... yet this interpretation is the most natural one, and the simplest procedure is to weaken the requirement that every line of a derivation be a logical consequence of the conjunction of its dependency set. What we may prove is that if a formula in a derivation contains no ambiguous names and neither do its premises, then it is a logical consequence of its premises. And this state of affairs is in fact intuitively satisfactory, for in a valid argument of use in any discipline we begin with premises and end with a conclusion which contains no ambiguous names.

We will see below how this idea eventually was played out.

With these nine choice points in mind, we turn to the elementary textbooks of the 1950s, where natural deduction was introduced to generations of philosophers and mathematicians. Different ones of these textbooks took different combinations of the choice points mentioned here, and this is why it is difficult to give a simple but adequate characterization of what natural deduction necessarily consists.

\section{Quine (and Rosser)}

The first publication of a modern-style natural deduction system is in Quine (1950a), where he says it is 'of a type known as natural deduction, and stems, in its broadest outlines, from Gentzen (1934/35) and Jaśkowski (1934)...' This textbook of Quine's was one of the main conduits of information about natural deduction to 'ordinary' philosophers and logicians, although as I will show below, there were also other pathways of a more technical nature. (There is also an article by Quine (1950b), which describes the textbook's method in more formal terms.) In the textbook Quine says the rule of conditionalization is 'the crux of natural deduction' (p. 166), and he 
points out that the metatheorem which we call 'The Deduction Theorem' $(\Sigma \cup\{\phi\} \vdash$ $\varphi$ iff $\Sigma \vdash(\phi \supset \varphi))$ is closely related to conditionalization but it has the 'status of [a] derived rule relative to one system or another. ${ }^{14}$

With regard to the first choice point in natural deduction proof systems, I have already remarked that Quine adopted Jaśkowski's book-keeping method to indicate relative scopes for subproofs, except that Quine's method of 'making assumptions' has one put a * next to the line number to indicate it is an assumption, and any line of the proof written after this assumption and before the use of a 'discharging rule' will also get $\mathrm{a}^{*}$ put next to its line number. When an assumption is made 'inside' an already active assumption, then it inherits a second *. Quine calls the $\supset$ I rule ' $\mathrm{Cd}$ '; it eliminates the most recent (undischarged) assumption in favour of a conditional using the formula that introduced the * as its antecedent and the line just prior to this conditional as its consequent. In this (1950b), Quine remarks that 'the use of columns of stars is more reminiscent of Jaśkowski's notation ${ }^{15}$ than Gentzen's. Its specific form is due to a suggestion by Dr. Hao Wang.' It is indeed very similar: Jaśkowski used a sequence of numerals to exactly the same purpose that Quine used a sequence of *. One difference was whether they were on the right (Jaśkowski) or left (Quine) of the numeral for the line number in the proof. Another difference, perhaps more important, concerned what happens when there are two separate subproofs both embedded within the same superordinate proofs. Jaśkowski indicates this by having the rightmost numeral in the sequence of subscope-indicating numerals be changed to a ' 2 ' in the second subproof, whereas Quine is restricted to using *s in both places, perhaps thereby allowing some confusion as to what the scope of a subproof is. It may also be that the possibility of confusion is heightened by the fact that Quine also does not employ any indication of what rules of inference are used to generate any given line in his proofs. On the other hand, since CD is the only way to end an embedded subproof in Quine's system, and since applying it eliminates the rightmost *, there will always be a line containing a conditional between any two independent subproofs that are at the same level of subproof embedding, and this line will have one fewer * than these subproofs. So therefore we can always tell when two subproofs are distinct, as Quine points out in (1950a, $156 \mathrm{fn} 1)$, using the terminology of 'an interruption of a column of stars'.

Concerning the other choice points for propositionallogic, Quine's system has no other subproof-introducing rules at all. Indeed, the only propositional rules are: 'introduce an assumption', $\mathrm{Cd}$, and his rule TF that allows one to infer any new line if it truth-functionally follows from the undischarged previous lines. Thus Quine has only one subproof-introducing rule, does not follow the int-elim pattern, and he does in effect allow all truth-functional implication tautologies as axioms by his use of TF (with the caveats I mentioned in the last section).

When it comes to the quantification rules, Quine's system contains both quantifiers rather than implicitly using negation and the universal quantifier to represent existential quantification. Quine says that not having existential quantifiers meant that

14 Suppes $(1957,29 \mathrm{fn}$ ) says that 'when the rule [of conditional proof] is derived from the other rules of inference rather than taken as primitive, it is usually called the deduction theorem.' Church $(1956,165)$ carefully distinguishes the deduction theorem (whether primitive or derived) from Gentzen formulations on the grounds that 'Gentzen's arrow, $\rightarrow$, is not comparable to our syntactical notation, $\vdash$, but belongs to his object language.' But Church is probably here thinking of Gentzen's sequent calculi (to be discussed in section 11 below) and not his natural deduction system.

15 By which Quine means the book-keeping method, not the graphical method. 
Jaśk owski did not have to worry about restrictions on a rule to eliminate $\exists$, and he said that Jaśkowski's restrictions on universal quantifier generalization were 'milder' (than Quine's). Quine had four rules for the quantifiers: introduction and elimination rules for each. As I discussed in the last section, Quine's rule of eliminating the existential quantifier does not introduce a subproof in the way that Gentzen's rule does. Quine's comment on this is that Gentzen's system 'had a more devious rule in place of [Quine's] EI, ${ }^{16}$ with the result that many deductions proved more complicated.' ${ }^{17}$

Here is a proof of a simple theorem, illustrating both the general style of Quine's proof system and also how he deals with the problems of variable interaction. (I have changed the symbolism to match Gentzen). Quine's method involved stating a preferred ordering on the entire infinite set of variables and requiring that UG always apply to a variable that is later in the ordering than any other free variables in the formula. ${ }^{18}$ Similarly, when using EI, the new variable must be later in the ordering than all the free variables in the formula. Whenever UG or EI is performed, the relevant variable is 'flagged' by writing it beside the annotation. There is a restriction on completed proofs that no variable may be flagged more than once in a derivation. And, as remarked in the last section, there is also a condition on 'finished proofs' that no flagged variable be free in the last line nor in any premises of the last line.

$\begin{array}{llll}* & 1 . & (\exists x)(\forall y) \text { Fxy } & \\ * & 2 . & (\forall y) \text { Fzy } & (1) z \\ * & 3 . & \text { Fzw } & (2) \\ * & 4 . & (\exists x) \text { Fxw } & (3) \\ * & 5 . & (\forall y)(\exists x) \text { Fxy } & (4) w \\ & 6 . & ((\exists x)(\forall y) \text { Fxy } \supset(\forall y)(\exists x) \text { Fxy }) & (1)-(5)\end{array}$

Quine's proof. $\mathrm{z}$ and $\mathrm{w}$ are later in the ordering than any free variables in their respective formulas, no flagged variable has been flagged more than once, and there are no free occurrences in the conclusion (nor in premises, since there are none). So the proof is correct as far as the flagging conventions are concerned. Note that line 1 introduced an assumption, and using it we generated line 5 . This then allows us to discharge the assumption and infer the conditional on line 6, which depends on no assumptions, since there are no *'s.

This presentation of natural deduction as an elementary proof method seems to be new to this work of Quine's; it does not appear in the third edition (1947) of his earlier Mathematical Logic. Quine does note the appearance of a system with rules of Conditionalization and EI in Cooley (1942, 126-140), although as Quine remarks, the

16 This is Quine's name for the rule Existential Instantiation. Since it eliminates an existential quantifier, it could be called $\exists \mathrm{E}$, except that I have reserved that name for the Gentzen-style rule that requires a subproof. Quine's use of the name EI should not be confused with 'existential quantifier introduction', which rule we are calling $\exists \mathrm{I}$.

17 Cellucci (1995) contains a very nice discussion of many of the issues concerning Quine's (1950a) system, especially those involving restrictions on variables and on how $\forall \mathrm{I}$ and EI interact. He also evaluates the plausibility of some of Quine's deviations from Jaśkowski and Gentzen.

18 In the first edition, that is. he changed his method in this regard in the second edition, where the ordering criterion is stated as 'it must be possible to list the flagged variables of a deduction in some order $v_{1}, \ldots, v_{n}$ such that, for each number $i$ from 1 to $(n-1), v_{i}$ is free no line in which $v_{i^{+}}, \ldots, v_{n-1}$ is flagged.' He retains the condition disallowing multiply-flagged variables (p. 164). 
EI rule is 'without exact formulation of restrictions.' ${ }^{19}$ Quine also says (p. 167) that explicit systems of this sort 'have been set forth by Rosser and independently by me in mimeographed lecture notes from 1946 on.' In the more technical (1950b), Quine comments more fully in fn. 3 about this matter, saying that he had mimeographed versions of the system in 1946 and 1948, but that the restrictions on variables in those systems were incorrect. ${ }^{20} \mathrm{He}$ also says:

I have lately learned that Barkley Rosser has had, since 1940, an exactly formulated system of natural deduction which perhaps resembles the present system more closely than any of the others cited above [by which he means Cooleys' and his own earlier work]. He set it forth in some mimeographed lecture notes in 1946-47. Having learned of this during travels in which I am still engaged, I do not yet know the details of his system. I was influenced in latter-day revisions of my present system, however, by information that Rosser's UG and EI were symmetrical to each other.

It is not at all clear what became of this work of Rosser's. He published his (1953) textbook, but this was not about natural deduction, neither mentioning the term nor referring to Gentzen or Jaśkowski. It is a textbook intending to show mathematician s how they might formalize their proofs of mathematical theorems. To this end Rosser introduced an axiom system for classical predicate logic, and then tried to show how mathematical reasoning could be represented in this system. But, as he remarks, this is not the way mathematicians actually carry out their proofs; and he goes on to illustrate how mathematical proofs could be imitated by a proof in the metalanguage of his axiom system. in this metalanguage he has the deduction theorem, and so he can represent the making of assumptions, and he can show how reductio proofs can be mirrored (this looks like the $\sim$ I rule mentioned at the beginning of section 4 above). When it comes to the quantifier introduction and elimination rules, these are also treated metalinguistically. (In the axiom system there is of course an axiom concerning universal generalization, but it does not work in the 'intuitive way' that a natural deduction rule does). In discussing the metalinguistic rule of EI (which he calls ' $C$ '), Rosser says it amounts to 'an act of choice' in generating 'an unknown, fixed' entity, and that this will explain the restrictions in using it (p. 128). The restrictions mentioned do not at all seem symmetrical to the restrictions on Rosser's rule of UG (which he calls ' $G$ '), which only require that the variable of generalization not occur free in any premises. But in any case Rosser does not in this book develop a system of natural deduction in the same sense that we have been discussing with Jaśkowski, Gentzen, and Quine.

Cellucci $(1995,314)$ reports that Quine met with Jaśkowski in Warsaw in 1933, and

19 Interestingly, Cooley says in his preface (p. viii) 'The text was originally written in mimeographed form for a course in which I collaborated with Prof. W. V. Quine of Harvard and I am greatly indebted to him for advice and stimulation.'

20 As I remarked two footnotes go, Quine changed the restrictions on UG and EI in the second edition of (1950a), which was published in 1959 with the remark 'In $\$ 28$ there are two convenient deductive rules that cannot be directly justified [namely, EI and UG], for the good reason that they serve to deduce conclusions from premises insufficient to imply them. In past printings of $\$ 28$ these rules have been indirectly justified by proving that deductions in which they are used will still turn out all right in the end, as long as certain arbitrary-looking restrictions are respected. In this new edition, $\$ 28$ is rewritten. The rules and restrictions are now explained and justified in a way that dispels the old air of artificiality.' Or so he says. 
helped with the translation of Jaśkowski's work into English. (I note that Jaśkowski (1934) was initially published in English.) Cellucci also reports that this was Quine's first exposure to natural deduction. We can see the background influence that Quine had from the very earliest statements of natural deduction (Jaśkowski's) through various pedagogical efforts of Cooley's (and Quine's own) in the 1940s, cumulating with the (1950a) publication and its various editions.

\section{Fitch}

About the same time that Quine was writing his textbook, Frederic Fitch was working on one too. It was published in 1952, and the method in it was also called 'natural deduction', marking thereby the second time the term was used in a work addressed to non-logicians. In the foreword of this book Fitch claims to have been using the method in teaching for the previous eleven years (p. viii), but in the preface he claims only that 'the fundamental ideas of this system of logic were conceived during... 1945-1946' (p. iv). In Fitch's mind the principal innovation of this system is 'the method of subordinate proofs' which, he says, 'vastly simplifies the carrying out of complicated proofs.' (p. vi). This method of subordinate proofs is 'suggested by techniques due to Gentzen (1934/35) and Jaśkowski (1934)' (p. vii).

Neither Quine nor Fitch refer to the other, even in later editions.

Quine and Fitch have made different turns at almost every one of the various choice points. To begin with, Fitch used Jaśkowski's graphical method to represent the subproof-structure of a derivation, rather than Jaśkowski's book-keeping method that Quine employed. The main difference between Jaśkowski's original method and Fitch's is that Fitch does not completely draw the whole rectangle around the embedded subproof. These are now generally referred to as 'Fitch diagrams'. I mentioned before that Jaśkowski's two methods were equivalent, and indeed seem almost identical in the propositional logic. But not only is Fitch's more clear when it comes to two successive subproofs at the same level, but also it seems that the logical restrictions one wishes to enforce for the quantifier rules can more easily be given by the method of boxes. Fitch 'flagged' subproofs with variables when they were introducing an assumption for existential quantifier elimination, and added some simple requirements on what formulas from an encompassing scope level can be reiterated inside such a flagged scope and which can be exported to outside scope levels when the embedded subproof is completed. As indicated in discussing the example proof in Quine's system, his method required that the annotation (justifications) of lines needed to be marked with the names of variables used when existentially instantiating and also when universally generalizing. There are then a set of restrictions on when formulas can be instantiated to which variables, and in addition there is the notion of 'an unfinished derivation', which is an otherwise good derivation except that some further restrictions on variables have not been observed in the construction of the proof ... but this is to be discovered afterwards, when inspecting the entire proof.

Another different turn is that Fitch adhered to the int-elim ideal: each connective comes with an introduction and an elimination rule, and there are no other rules of inference. (In particular, there are no axioms or lists of equivalences or rules that implicitly appealed to axioms or equivalences.) And as a pedagogical point, Fitch cited both the rule of inference employed and the line numbers of the precondition-formulas as a part of the annotation. Quine did not use rule-names. Fitch had subproof- 
requiring rules for $\supset \mathrm{I}, \vee \mathrm{I}$, and $\exists \mathrm{E}$. The fact that Fitch had the rule of $\exists \mathrm{E}$ rather than Quine's EI has the many ramifications of the sort outlined above in section 4. Fitch does not employ parameters, but uses variables exclusively. ${ }^{21}$

A proof in Fitch's system for the theorem proved above in Quine's system would look like this. (Fitch uses 'uq' and 'eq' for $\forall$ and $\exists$ in the annotations).

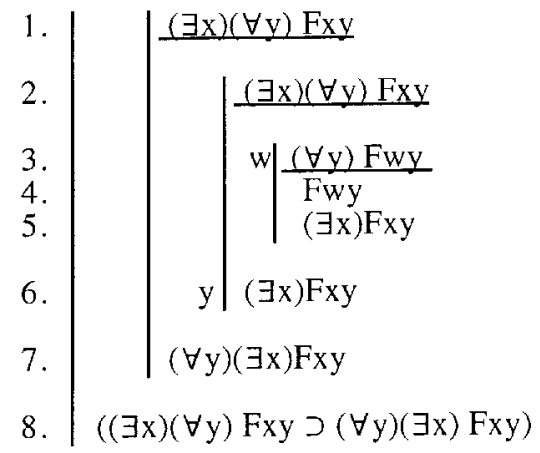

hypothesis

1, Reiteration

hypothesis

3, uq elim

4 , eq int

2, 3-5, eq elim

$2-6$, uq int

1-7, $\supset$ int

Fitch's proof. Line 3 is the 'arbitrary' instance of line 2 , to be used in showing that line 5 follows from this arbitrary instance. The fact that it is 'arbitrary' is enforced by the $w$-scope line, prohibiting the use of $w$ in other formulas. Since line 5 follows from the winstance, line 6 follows from the quantified formula on line 2. The rule of universal generalization is applied to this line, using the variable $\mathrm{y}$, and exporting out one scope level. Line 8 is the desired conditional. Each scope level starts with a hypothesis, the formula above the horizontal line. The fact that line 8 is proved with no hypotheses shows it to be a theorem.

\section{Copi (and Rosser)}

The most enduring and widely-used textbook from this era is Copi's (1954). This book does not refer to either of Quine's or Fitch's earlier books, and says only that the quantifier rules "are variants of rules for "natural deduction" which were devised independently by Gerhard Gentzen and Stanisław Jaśkowski in 1934' (p. 76). There are no further references to any published work on natural deduction, although the preface says that some of the material 'was first communicated to me by ... Professor Atwell Turquette' and that 'the logistic systems [axiomatic systems] R.S. and RS , $_{1}$ which are set forth in Chapters 7 and 9, respectively, are early versions of calculi which appear in revised form in Professor Barkley Rosser's [1953]. ${ }^{222}$

21 Although it is true that Fitch doesn't distinguish parameters from variables in the way that Gentzen does (and some later writers do)... which is the point of the sentence in the text... it might also be noted that, officially, Fitch doesn't have any free variables. They are all thought of as 'names' - not 'dummy names', but real ones. Although this seems not to have any logical force in elementary logic, it feeds into his later interest in the substitution theory of quantification.

22 I continue here with the puzzle that Quine said that Rosser had developed natural deduction systems, and yet there are none such in Rosser (1953). From the fact that Copi had taken material from earlier versions of Rosser's (1953) and that this material consisted only of axiomatic developments, it seems plausible to suppose that there never was any natural deduction in the early Rosser manuscripts... except, as mentioned above, in some sort of metalinguistic sense. For, Copi would certainly have mentioned it, or used it, given his fulsome acknowledgements of Rosser. Further, according to Copi (pp. ix $-\mathrm{x}$ ), Rosser read the manuscript of Copi's book, and (apparently) made no claims about priority in the development of natural deduction. Rosser and Turquette are, of course, 
Copi uses a version of Jaśkowski's graphical method, although not quite like Fitch's. He indicates the embedded subproof by means of drawing a 'bent arrow ... with its head pointing at the assumption from the left, its shaft bending down to run along all steps within the scope of the assumption, and then bending inward to mark the end of the scope of that assumption.' (See the example proof below.) Unlike Quine's system, Copi's does not have a general rule of 'follows by truth functional logic' but instead has a set of nine 'elementary valid [propositional] argument forms,' to go along with his rule of conditional proof (' $\mathrm{CP}$ '). But unlike Fitch, Copi did not organize these argument forms into the Gentzen ideal of introduction and elimination for each connective. He does have int-elim rules for some of the connectives: the conditional (calling $\supset \mathrm{I}$ ' $\mathrm{CP}$ ' and calling $\supset \mathrm{E}$ by its traditional name, 'MP'), the conjunction (calling \& E 'Simp' and \& I 'Conj'), as well as for the quantifiers. For disjunction Copi has a normal $\vee$ I rule (called 'Add'), but he uses $\mathrm{DS}^{23}$ to handle some of $\vee \mathrm{E}$, while other parts of $\vee \mathrm{E}$ are dealt with by CD. ${ }^{24}$ Copi also has MT ('Modus Tollens': from $(\varphi \supset \gamma)$ and $\neg \gamma$ infer $\neg \varphi)$, and a 'Destructive Dilemma' that uses MT and disjunction where CD used MP and disjunction. ${ }^{25}$ In addition, Copi had a list of 'logical equivalents' which 'may be substituted for each other wherever they occur,' thus in effect allowing axioms. Despite deviation from Gentzen's system due to the lack of introduction and elimination rules for some of the propositional connectives, and the presence of rules that are not in this format, and the existence of 'equivalents', his text was so popular that this system became the prototypical example of a (propositional) natural deduction system.

Copi, like Quine and unlike Fitch/Gentzen, does not employ a subproof when eliminating existential quantifiers. They are instead eliminated directly in the same proof level as the existentially quantified formula ... and thus is a Quine-system, in the sense mentioned in section 4 . He has an appealingly simple restriction on the variables introduced by this EI rule: the variable used to state the instance has to be new to the proof (as well as substituted properly, of course). But this makes the restrictions on variables employed in universal generalization become more difficult. Besides the usual proper substitution requirements, Copi states these other requirements as "[the free variable to be generalized upon] does not occur free either in any propositional function inferred by EI or in any assumption within whose scope [the formula being generalized] lies.'

Like Quine, Copi altered the statement of the restrictions, although in Copi's case (unlike Quine's) it was because they were logically incorrect. In Copi's second edition (1965) they are replaced by a method described in Copi (1956), which changed the restrictions on variables cited in universal generalization. ${ }^{26}$ No longer is it merely "the free variable to be generalized upon' that can't occur free if it was somewhere inferred

long-time collaborators, especially in the field of many-valued logics. And so it is possible that they 'doodled around' with systems of natural deduction informally, and that Turquette then forwarded these ideas to Copi.

23 For 'Disjunctive syllogism' Copi used the traditional names for his rules of inference. This rule is sometimes called MTP ('Modus Tollendo Ponens') or 'unit resolution' : from $\sim \Phi$ and $(\Phi \vee \Psi)$ infer $\Psi$ [and a symmetrical form for eliminating from the other side of the disjunction].

24 For 'Constructive Dilemma'. This is the rule I called Separation of Cases above. Recall that this differs from the usual $\vee$ E rule in not requiring subproofs but instead merely requiring the conditionals to be at the same subproof level as the disjunction, i.e. from $(\varphi \supset \gamma)$ and $(\sigma \supset \theta)$ and $(\varphi \vee \sigma)$ infer $(\gamma \vee \theta)$.

25 That is, from $(\varphi \supset \gamma)$ and $(\sigma \supset \theta)$ and $(\neg \gamma \vee \neg \theta)$ infer $(\neg \varphi \vee \neg \sigma)$.

26 There was also a change in the third printing of the first edition, in 1958. However this change, which seems adequate, did not survive when the second edition came out in 1965 where it was replaced by the 1956 method. 
by EI, but now it is ' any free variable in the formula being generalized upon' that can't occur free if it was introduced by EI in any formula. The original error showed that the completeness / soundness proofs of the original system were flawed, and they are altered in both (1956) and in the second edition (1965). Copi (1956) says that these errors were ' pointed out to me by Professor Donald Kalish'. Ironically, Kalish (1967) also shows that these (1956) changes, which are carried over to the second edition, are also inadequate. ${ }^{27}$

A proof in Copi's system of the example we have been following is:

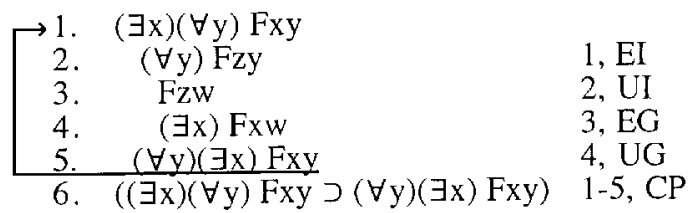

Copi's proof. After completing the proof we need to check whether the application of EI introduced a new variable (it did) and whether the w of line 4 was a variable introduced by EI (it wasn't). The fact that line 1 introduced an assumption and from it we generated line 5 , is indicated by the arrow and lines, which discharges the line 1 assumption and we infer the conditional on line 6 .

\section{Suppes}

Suppes (1957) seems to be the next of the natural deduction textbooks to appear, and although it appeared in 1957, the preface says that it was initially a joint book with J. C. C. McKinsey, who 'wrote the original drafts of Chapters 9, 10, and 11.' Since McKinsey died in 1953 the book must have been well underway before the appearance of Copi (1954). The preface also remarks that there were 'several preliminary editions used in courses since the summer of 1954.' Suppes does not refer to Gentzen or Jaśkowski, nor to Rosser; and there is but one passing reference to Quine (1950a) and to Copi (1954) on p. 81, obviously added as toss-away references immediately before the publication of the book. In section 3 above I pointed out that Suppes's method of representing the structure of subproofs was different from the three classic methods developed by the founders of natural deduction, Jaśkowski and Gentzen. And I mentioned that those who might have initially been attracted to Jaśkowski's bookkeeping method adopted Suppes's superior method instead.

Like Quine and unlike Fitch or Copi, Suppes has a rule ' $\mathrm{T}$ ' which allows one to 'introduce a sentence $S$ in a derivation if there are preceding sentences in the derivation such that their conjunction tautologically implies S.' Recall that when making an assumption in this system, the line number is placed into set braces. when applying the rule $\mathrm{T}$ of tautological implication (or any other rule except for conditional proof), one indicates the set of all premises upon which that line depends by forming the union of all premises that the lines appealed to by this use of T depended upon, and placing the result to the left of the line number. As usual, the rule of conditional proof (CP) generates a new line that has a conditional as its main connective, has some formula

27 See also Parry (1965), Leblanc (1965), and Slater (1966). In the third edition (1967), Copi changed from a Quine system to a Fitch-Gentzen system, presumably on the grounds that it is much easier to state the restrictions on variables. Kalish (1974) claims that the new system is correct, but he also shows that the proof of its soundness continues to be incorrect. 
that was introduced by the assumption rule as an antecedent, and has some later-inthe-proof formula as its consequent. The set of formulas that this conditional depends upon is: those that the consequent depended upon minus the line number of the antecedent. Suppes's rule T in effect allows axioms in the system, in the same sense as Quine's TF rule does. Suppes introduces a 'derived rule' of $\sim$ I to the propositional logic system, calling it 'Reductio', and using it extensively in his examples. Like the $\supset$ I rule, Reductio requires a subproof as a precondition of its application.

Another innovation of Suppes was to employ the notion of ambiguous names to aid with the statement of the quantifier rules. He says that 'the technical device used here [is] apparently new in the literature of first-order logic' (p. 81), but that 'the central idea of this approach is related to Hilbert's $\varepsilon$ symbol' as set forth in Hilbert and Bernays (1934/1939). The idea there was that ' $\varepsilon x F x$ ' was to designate 'an arbitrary or indefinite F' about which nothing was known other than that it was F. Suppes's method was to have an orthographically distinct category of these ambiguous names, separate from the variables and from the constants ('proper names'). He used Greek letters for these arbitrary names, while the variables were roman letters from the end of the alphabet and the constants were roman letters from the beginning of the alphabet. In this way an existential quantifier was to be eliminated (called Existential Specification, ES) by replacing it with a one of these ambiguous names, so long as it is new to the proof. ${ }^{28}$ ES does not require an embedded subproof, and so this system is a Quine-system in the sense described above. Existential Generalization (EG) can be the result of generalizing on either a proper or an ambiguous name, or on a variable (these three types form the category of 'term'). Universal Specification (US) can replace its variable of generalization by any term. Finally, Universal Generalization (UG) can occur only on a free variable and then only if that variable is not 'flagged' (and only if the variable does not occur as a subscript to any arbitrary name in the formula - see the last footnote). Suppes uses the notion of a 'flagged variables' as an aid to stating the UG rule, requiring that any premise of the argument that has a free variable must have that variable flagged, which means to indicate any such variable on the line in the proof where that premise is entered. (Suppes does this by writing the variable alongside the justification for the line). Any line that appeals to a line with a flagged variable in its justification will also have that variable flagged. UG cannot apply to a flagged variable.

Proofs in Suppes's system look very much like those in Quine's, except for (a) the appearance of ambiguous names, (b) the notion of subscripting variables, (c) the

28 As with any of the systems we need to make sure that the substitution is proper. Suppes chooses to do this as a matter of conditions on the proper application of rules, rather than giving a separate definition of 'proper substitution' and then requiring the rules to embody proper substitution. For example, suppose that from $(\forall x)(\exists y)$ Fxy we infer Fx $\alpha$ by US and then ES ( $\alpha$ being an ambiguous name). We want to prevent inferring $(\exists \mathrm{x})$ Fxx from this last formula by EG. One way to do this (followed by Quine, Fitch, and Copi) is to say that Fxx is not a proper substitution instance of Fxo (or perhaps conversely), and this notion of proper substitution is a restriction on using any of the quantifier rules. But Suppes instead decides to say that 'when an ambiguous name is introduced by ES it is to include as a subscript all the free variables occurring in the original formula'. So, in our example the ES rule would have generated $F x \alpha_{x}$. And now there is a restriction on EG that one may not apply an existential quantifier to a formula using a variable which occurs as a subscript in the formula. Similar strategies are employed in US as well as EG. The notion is also appealed to in UG, but here the background reason is different and can't be dealt with merely on the basis of a proper substitution. it is used to prevent universal generalization on a variable when the formula still has an ambiguous name in it that was introduced after a universal specification. Those familiar with resolution system will see all these restrictions as ways to recognize skolem functions and to deal correctly with them. 
explicit marking of which formulas are current premises for each line, and (d) the consequent lack of other scope-indicating methods such as *'s. The proof we have been tracking would look like this:

\begin{tabular}{|c|c|c|}
\hline & $\begin{array}{ll}\text { 1. } & (\exists x)(\forall y) \text { Fxy } \\
\text { 2. } & (\forall y) \text { Fay } \\
\text { 3. } & \text { Fay } \\
\text { 4. } & (\exists x) \text { Fxy } \\
\text { 5. } & (\forall y)(\exists x) \text { Fxy } \\
\text { 6. } & ((\exists x)(\forall y) \text { Fxy } \supset(\forall y)(\exists x) F x y)\end{array}$ & $\begin{array}{l}\text { P } \\
1 \mathrm{ES} \\
2 \mathrm{US} \\
3 \mathrm{EG} \\
4 \mathrm{UG} \\
(1)-(5) \mathrm{C} .\end{array}$ \\
\hline
\end{tabular}

Suppes's proof. The ambiguous name $\alpha$ is introduced by ES. There are no premises that contain free variables, so there are no flagged variables. And there is no use of ES to a formula with free variables in it, so there are no subscripted variables. Line 1 introduces a $\{1\}$ to indicate it is an assumption or a premise. Each of the lines $2-5$ was inferred from a line that had this dependency, and thus they all do also. The dependency is discharged when using conditional proof on line 6 , and now there is an empty set of dependencies, showing line 6 to be a theorem.

\section{The choice points revisited}

As we have seen, in the fundamental textbooks that introduced natural deduction to the 'average philosopher', there were many problems with the precise statement of the natural deduction rules for the quantifiers, and we've also seen that the Gentzen ideal of introduction and elimination rules for each connective was followed only by Fitch. Nonetheless, these four systems form the early history of natural deduction as taught to generations of philosophers and mathematicians, and were the acknowledged source of the next wave of elementary logic textbooks, which seem to have overcome the difficulties in stating the quantifier rules and also seem to have settled on not having introduction and elimination rules of the Gentzen style for the propositional connectives. This next wave includes Anderson and Johnstone (1962), Kalish and Montague (1964), Lemmon (1965), Mates (1965), and Pollock (1969), as well as later editions of the texts mentioned earlier.

In summarizing the fate of our nine choice points - that is, how they were taken up by later textbooks, both the second wave of 1960s texts and the third wave of 1970s-1980s texts-we would come to these conclusions:

I. Propositional proof-style is about equally divided between the Jaśkowski's graphical method and Suppes's method.

II. About half the texts 'allow axioms' in the sense of having sets of equivalents or implications and a primitive rule that allows replacement of a line (or part of it) by an equivalent. Pretty much all the other texts have such a rule as a derived rule.

III. Very few texts in the second and third wave maintained the ideal of int-elim rules for all the propositional connectives and no other rules. (Of the second wave mentioned above, only Anderson \& Johnstone maintained this intelim ideal). Most textbooks have a set of elementary propositional rules, rather than a general 'truth functional inference' rule. 
IV. All texts have a primitive subproof-requiring rule $\supset$ I; almost all texts have a primitive subproof-requiring rule of $\sim \mathrm{I}$ or $\sim \mathrm{E}$ (that is, some form of reductio); most texts have a subproof-requiring rule of $\vee \mathrm{E}$, although a strong minority use SC (separation of cases) instead; about half the systems that have a material equivalence $(\equiv)$ have a subproof-requiring rule for $\equiv$ I.

V. Except for Mates (1965), all authors had primitive universal and existential quantifiers; and even Mates introduced the existential quantifier as a derived symbol and gave it appropriate int-elim rules.

VI. Somewhat more than half of our authors have employed the $\exists \mathrm{E}$ (subproofrequiring) version of existential quantifier elimination, while the remainder use a version of Quine's EI that does not require subproofs.

VII. Most authors do allow free variables in premises and conclusions. About half treat them semantically as existentially quantified and the other half as universally quantified.

IX. The ploy of employing a separate category of 'arbitrary names' or 'parameters' was not widely adopted.

\section{Advanced works: Bernays, Feys, Church, Kleene, and Curry}

The works surveyed in the previous sections were all introductory textbooks, and I discussed them so as to show why the term 'natural deduction' has come to indicate the types of systems now in the front of the mind of philosophical logicians, most of whom have taken their elementary logic from these textbooks or later ones patterned after these. In addition to the elementary textbooks, there were a number of writings at a high level of sophistication which occurred between the 1934 inception of natural deduction and the widespread acceptance of natural deduction in the late 1950s. The present section aims to cite some of the most influential works of this nature, and to explain why they did not in fact have much of an impact on the 'ordinary philosopher.' The underlying reason for this is that these works were too advanced and complex for these 'ordinary philosophers' to comprehend themselves, and the works did not get explained to them by anyone capable of understanding them. For example, the writers of the first wave of textbooks might have explained these works in a 'preface for the instructor', for example, or at least have referred to these works as being worthy of further study. But in fact, of the four textbook authors canvassed above, only Suppes refers to any of the works I am about to discuss, and he does not refer to the relevant area. See Suppes, 1957, 70.

The authors I have in mind here, those authors who first read Gentzen and Jaśkowski and who followed up this natural deduction methodology by writing further important expository and exploratory works in this area, are Bernays (1936), Feys (1946), Curry (1950), Kleene (1952) and Church (1956). Of these five authors, Curry's work on natural deduction was much more influential than any of the other's work on natural deduction. For, even within the realm of practicing logicians the latter four works had a muted impact so far as Gentzen's natural deduction is concerned. Bernays's work was in the form of mimeographed notes taken at his Princeton lectures. These very interesting notes show that Bernays was aware of the relationship between Hilbert's $\varepsilon$-term operator and the use of 'arbitrary names' in the rules of $\exists$ elimination and $\forall$-introduction (see p. 73ff). It also gave a quite detailed account of 
Gentzen's natural deduction, although it was rather skimpy on actual proofs in the system. Even though these notes were widely distributed, the fact is that they covered many different topics in addition to an explication of Gentzen's natural deduction systems; and many of these differing topics appeared in other published works of Bernays that did not emphasize the natural deduction aspect but rather concentrated on metalogical topics. It was often these other topics that readers of Bernays found most interesting, and so these readers naturally were more comfortable in reading his works that did not explicate natural deduction. Further, in the realm of logical systems, Bernays was mostly interested in intuitionistic systems and their properties. Many logicians, especially American ones, did not share this interest and did not follow his works carefully (although it is cited in Church 1956, 215n365 and also in Curry 1950 at many places).

Feys (1946) was in French, which set it apart from the majority logic works that were in English or German, and also Feys's interests were always more in the realm of modal logics, culminating in Feys (1965) in which the natural deduction aspects were much subordinated to the modal logic. For these reasons Feys's work on natural deduction did not have much impact (only Curry 1950 refers to it, among the writers here surveyed).

Church's monumental work - which enjoyed a very wide circulation in preliminary versions during the 1940s and early 1950s_-does not actually discuss natural deduction systems very much. In the pedagogical body of the book his interest is in teaching an axiomatic system and with proving properties of this system. In the amazingly thorough historical parts of this book the main discussion of natural deduction as an independent manner of describing logic occurs on pp. 164-165 where he says

The idea of using the deduction theorem as a primitive rule of inference in formulations of the propositional calculus or functional calculus [first order logic] is due independently to Jaśkowski and Gentzen. Such a primitive rule of inference has a less elementary character than is otherwise usual, and indeed it would not be admissible for a logistic system according to the definition as we actually gave it [as an object-language logical system]. But this disadvantage may be thought to be partly offset by a certain naturalness of the method; indeed to take the deduction theorem as a primitive rule is just to recognize formally the usual informal procedure (common especially in mathematical reasoning) of proving an implication by making an assumption and drawing a conclusion.

However, Church also says (p. 94n181), 'Advantage s of the deduction theorem in this role are largely psychological and heuristic.' Presumably he means that there is no 'real' logical reason to adopt the deduction theorem as a primitive rule, and therefore no 'logical' reason to employ natural deduction systems. And so, although Gentzen's natural deduction is mentioned by Church and although this book was known by virtually every logician even before it was finally published, the topic was given so short a shrift that it certainly cannot be said to have encouraged the study of natural deduction by many people. And it certainly seems to have had no impact at all on the early teaching of natural deduction.

Kleene's (1952) advanced textbook contains a substantial discussion of Gentzen's sequent calculus in chapter 15 (the last chapter). However, this book contains no discussion of Gentzen's natural deduction method, and I will show in the next section that these two methods are quite different from each other. Furthermore, this final 
chapter of Kleene's textbook is seen, even by Kleene, as only rarely being reached in classroom use (pp. v-vi):

The book is written to be usable as a text book by first year graduate students in mathematics (and above)... The author has found it feasible to complete [the first] ten chapters... in the semester course which he has given... The remaining five chapters can be used to extend such a course to a year course, or as collateral reading to accompany a seminar.

I think it is true that many logicians became aware of Gentzen's work through its being mentioned in Kleene's book. However, none of these readers will have learned anything about natural deduction from the book, for it is not at all discussed. Those readers who were interested would have been sent to the original source and would have discovered for themselves what Gentzen meant by natural deduction. Part of the reason that they might have become interested is due to Kleene's sections 23 and 24 (pp. 98-107) which contain a discussion of 'Introduction and elimination of logical symbols.' Here Kleene provides 'a collection of derived rules' starting with the deduction theorem (renaming it now ' $\supset$-Introduction') and including int-elim rules for all the connectives and quantifiers. They are stated in this form (the example is $V$ Elimination):

\section{If $\Gamma, \mathrm{A} \vdash \mathrm{C}$ and $\Gamma, \mathrm{B} \vdash \mathrm{C}$, then $\Gamma, \mathrm{A} \vee \mathrm{B} \vdash \mathrm{C}$}

Here we can see that this rule is treated as a metalinguistic rule that guarantees the existence of a certain proof (in the then clause) if there are proofs of the sort mentioned in the if clause. Kleene shows that these rules are all derivable, and hence admissible, by showing how to ' unabbreviate' them in favour of an explicit sequence of steps in the axiomatic system he has developed. So while these sections of the book illustrate the strategy of natural deduction proofs, and incidentally discuss how the restrictions on the variables should go, there is no independently-developed system that embodies natural deduction. But those readers who might be attracted to this sort of logical system would most likely have been directed to Gentzen's original articles and would have found it congenial.

Curry (1950) is a set of five lectures given at Notre Dame University in 1948. 'The book aims to give a self-contained account of the approach to the logical calculus by means of the inferential rules as given by Gentzen in his thesis (Gentzen 1934/35) ... In regard to the expository aspect, I share in the opinion that the inferential rules of Gentzen and Jaśkowski form one of the most natural and fruitful approaches to the propositional and predicate calculuses' ( $\mathrm{p}$. iii). But the book is not elementary: 'The exposition attempted here is intended for mature persons... it is assumed that the reader can cope with mathematical arguments of considerable generality and abstractness...' (p. iii). Curry says that he has come to believe, on the basis of his investigations into combinatory logic, 'that Gentzen's L-system [the sequent formulation] is really a more profound and, in a sense, a more natural approach than the T-system [Curry's name for the natural deduction N-system].' This perhaps means that the exposition was of more interest to those researchers interested in the metatheoretic properties of L-systems than in natural deduction. (There are in fact only a very few object-language proofs given in the book. It would be very difficult to achieve any expertise in natural deduction merely from looking at the examples.) 
Despite the fact that these lectures were merely printed from typescript, they apparently had quite a wide distribution and have been cited by a large number of authors. Curry continued to be a champion of (what he came to call) 'the inferential calculus' of Gentzen ${ }^{29}$ (so called because the basic items were statements of valid inferences, rather than axioms.)

My contention is, after examining the works of the authors mentioned in this section, that they played only a minor role in the formation of the stereotype of natural deduction. Instead, they kept alive the formal virtues of the sequent-calculus formulations of Gentzen, and succeeded in drawing the attention of some logicians of the 1940s and 1950s to the Gentzen way of proceeding. But as for establishing the notion of natural deduction in the minds of the philosophical-mathematical community, it was the elementary textbooks that did that.

\section{Gentzen's sequent calculus}

I have already mentioned that some researchers who became interested in 'Gentzen's logical methods' were in fact more attracted to his sequent calculi than to his natural deduction calculi. But this is sometimes obscured because the distinction between the two proof systems is not fully appreciated, and it might be thought that anyone interested in Gentzen's logical systems was thereby interested in his natural deduction logics.

In his 1934/35 article, Gentzen in fact spent much more time developing his Lcalculi than he did his natural deduction logics. These L-logics were presented in a format known as the sequent calculus, which Gentzen presented in a tree format but where the elements at nodes are sequents rather than simple formulas (as was the case in Gentzen's presentation of natural deduction proofs). A sequent is anything of the form $\Phi_{1}, \ldots, \Phi_{\mathrm{n}} \rightarrow \Psi_{1}, \ldots, \Psi_{\mathrm{k}}$ where the $\Phi_{\mathrm{i}}$ and $\Psi_{\mathrm{i}}$ are ordinary formulas (and either of $\mathrm{n}$ and $\mathrm{k}$ could be 0$){ }^{30}$ The interpretation of this notation is a matter of some dispute, but a natural understanding is that a sequent asserts the existence of a proof of one of the $\Psi_{i}$ 's from the set of premises $\left\{\Phi_{1}, \ldots, \Phi_{n}\right\}$. Equivalently one could say that it asserts the existence of a proof from the conjunction of the $\Phi_{i}$ 's to the disjunction of the $\Psi_{i}$ 's. Gentzen says it is to be interpreted as equivalent to $\left(\left(\Phi_{\mathrm{i}} \& \ldots \& \Phi_{\mathrm{n}}\right) \supset\left(\Psi_{1} \vee \ldots \vee \Psi_{\mathrm{k}}\right)\right)$, presumably meaning equivalent to this formula's being a theorem. There are three types of rules of inference in the L-calculi: one is that all branches of a proof tree start with $\Phi \rightarrow \Phi$ sequents, and the other two types of rules describe how to extend a proof tree by altering a sequent into another one. These other two types of inference rules are called the 'structural' and the 'operational' rules, the former not doing anything to the logical symbols in a proof but only to the structure of the sequents (such as saying that extra premises can be added to a valid argument, or permuting their order; additionally there is a special rule, 'cut'). The operational rules, are always introduction rules for the connectives, but divided into whether the connective is introduced on the left or the right of the $\rightarrow$. So for each connective there will still be two rules, but now rather than being elimination and introduction they are introduction on the left and introduction on the right.

29 As evidenced by his (1960/61), the textbook (1963), and various expository articles such as his (1965).

30 Gentzen also remarks that the natural deduction logics could be formulated with sequents, and then the difference between the N-systems and the L-systems is that the N-systems have k (the number of 'conclusion formulas') be 0 or 1 . 
These L-systems are naturally interpreted metalinguistally. The rules of inference amount to saying 'If there is a proof that looks like such-and-so, then there is a proof that looks like so-and-so.' And a proof tree in an L-system in essence says : 'Granted that there are always proofs of $\phi$ from $\phi$, here's a demonstration that there must also be a proof of $\Psi$ from the set of premises $\Delta$.' This suggests that $\rightarrow$ is profitably seen as meaning $\vdash$, although not everyone agrees with this. Church $(1956,165)$, for example, says $\rightarrow$ cannot be interpreted as the metalinguistic $\vdash$ because it is in Gentzen's object language. ${ }^{31}$

I will here give a derivation in LJ sequent calculus for the simple theorem $\sim \exists \mathrm{xFx} \supset \forall \mathrm{y} \sim \mathrm{Fy}$. That this is a theorem is shown by its being the only formula on the right side of an $\rightarrow$ which has no formulas on its left side. I have not given all the rules of inference for a sequent calculus, but will trust that citing structural rules like 'interchange' will be clear (it permutes the order of elements), that citing the rule 'cut' is obvious (it allows one to 'piece together' two derivations where the conclusion of one derivation is a premise of another), and that the reader can follow rules like $\forall$ I$\mathrm{R}$ (universal quantifier introduction in the right) and $\sim \mathrm{I}-\mathrm{L}$ (negation introduction on the left). A sequent below a line is justified by the rule cited beside it on account of the sequent(s) above that line.

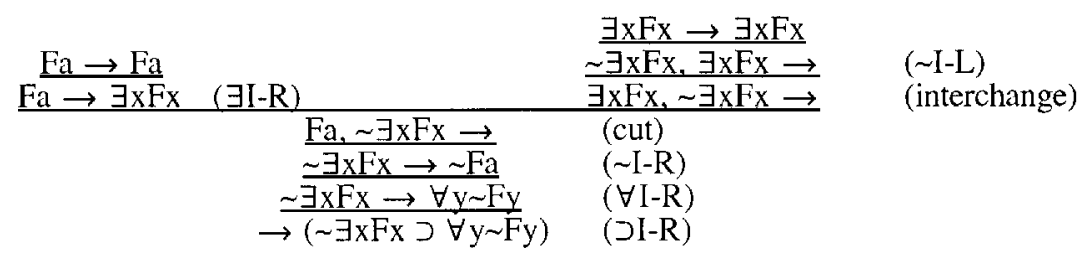

It can be seen that each branch of the tree starts with the assertion that there is a proof of a formula from itself, than various rules alter these statements until the two branches can be unified by an application of Cut. Then further modifications are made until we arrive at a sequent with no premises and the desired formula as the sole conclusion.

One might wonder why Gentzen bothered to introduce the rather unnatural Lsystems in addition to the N-systems. Here's what he says:

A closer investigation of the specific properties of the natural calculus finally led me to a very general theorem which will be referred to below as the Hauptsatz. The Hauptsatz says that every purely logical proof can be reduced to a definite, though not unique, normal form. Perhaps we may express the essential properties of such a normal proof by saying: it is not roundabout. No concepts enter into the proof other than those contained in its final result, and their use was therefore essential to the achievement of that result...

In order to be able to enunciate and prove the Hauptsatz in a convenient form, I had to provide a logical calculus especially suited to the purpose. For this the natural calculus proved unsuitable... [T]herefore, I [developed] a new calculus of logical deduction possessing all the desired properties in both their intuitionist and

31 Prawitz $(1965,91)$ puts the point like this: 'A proof in the calculus of sequents can be looked upon as an instruction on how to construct a corresponding natural deduction.' 
their classical forms (' $\mathrm{LJ}$ ' for intuitionist, ' $\mathrm{LK}$ ' for classical predicate logic). The Hauptsatz will then be enunciated and proved by means of that calculus... In section $\mathrm{V}$, I prove the equivalence of the logical calculi NJ, NK, and LJ, LK, developed in this paper, by means of a calculus modeled on the formalisms of Russell, Hilbert, and Heyting.

The equivalence that is shown is that the same theorems are probable. He thus shows that anything provable in the axiomatic theory can be proved in each of the $\mathrm{N}$ - and Llogics, and that a proof in either of these logics can be simulated in the axiomatic theory. ${ }^{32}$

Gentzen proved that for any formula $\phi$ provable in a N-system, there is a proof tree in the corresponding L-system that has the sequent $\rightarrow \phi$ as its root, i.e. $\vdash \phi$. And for each sequent $\Phi_{1}, \ldots, \Phi_{\mathrm{n}} \rightarrow \Psi_{1}, \ldots, \Psi_{\mathrm{k}}$ that occurs as the root of an L-proof tree there is a proof of $\left.\left(\left(\Phi_{1} \& \ldots \& \Phi_{n}\right)\right) \mathrm{k} \supset\left(\Psi_{1} \vee \ldots \vee \Psi_{k}\right)\right)$ in the corresponding $\mathrm{N}$-system. But of course N-proofs and L-proofs are quite different: Using Gentzen's methods for the Nsystems we operate directly with the object language formulas and construct a proof tree starting with assumptions, whereas in the L-systems we start with 'trivial proofs' and show that there must exist more and more complex proofs until we have shown that there is a proof of the formula we are interested in from the premises we are given.

The sequent formulation of the $\mathrm{N}$-systems therefore is not natural deduction system. It is a metalinguistic proof system, and automated theorem proving programs (as well as people) who use such a formulation to construct proofs (more accurately, to construct proofs that there is a proof) are not employing natural deduction as laid out by Gentzen (or Jaśkowski). It may be that there are many nice properties of Lproofs, such as there always being a cut-free proof for any sequent and therefore such proofs always obeying a subformula property. But against this we should look back to the advantages Gentzen claimed for natural deduction proofs and at the method by which he achieved these advantages. And we see that sequent proofs do not embody these advantages, and they fail to embody them precisely because they are not proofs in a natural deduction format. ${ }^{33}$

\section{Concluding remarks}

I started this story with the astonishing occurrence of two independent researchers happening upon the identical logical methodology at essentially the same time. 1934 was a propitious year - at least from a logical point of view. The story continued with

32 As if this weren't already enough for one article, Gentzen also gave a decision procedure for intuitionist propositional logic and 'a new proof of the consistency of classical arithmetic without complete induction.'

33 Further, it would be wrong to let stand unchallenged Gentzen's belief that natural deduction systems do not also have the same nice metatheoretical properties that the sequent calculi have, or that it is somehow much harder to prove them for natural deduction systems than for sequent systems. Prawitz's (1965) landmark work showed that natural deduction systems in fact have a Hauptsatz and all the other properties of sequent systems. And with the correct formulation, they are even easier to prove for natural deduction systems. Here's what Prawitz says $(1965,7)$ : ' The result that every natural deduction can be transformed into this normal form is equivalent to what is known as the Hauptsatz or the natural form theorem, a basic result in proof theory, which was established by Gentzen for the calculi of sequents. The proof [in the present work] of this result for systems of natural deduction is in many ways simpler and more illuminating.' Allen Hazen remarks, in personal communication, that 'to give Gentzen his due, Prawitz only proves his normalization theorem for intuitionisticlogic, and for the $V$ free and $\exists$-free classical fragment. Proving it for the full classical logic is much harder.' 
these discoveries going 'underground', being known only to the logical sophisticates, and being passed from one enlightened initiate to another by mimeographed lecture notes and preprints. But then came the early 1950s when the method became a part of some elementary textbooks; and so popular were these textbooks that within a very short period of time indeed this method of doing logical proofs became the only way that the vast majority of philosophers had even heard of. The different directions taken by the different authors on various of the details have given rise to a panoply of systems that are linked mainly through their common history.

And this was a Good Thing. ${ }^{34}$

\section{References}

Anderson, A. and Johnstone, H. 1962. Natural Deduction, the Logical Basis of Axiom Systems, Belmont, CA: Wadsworth.

Anellis, I. 1991. 'Forty Years of "Unnatural" Natural Deduction and Quantification: A History of FirstOrder Systems of Natural Deduction from Gentzen, to Copi'. Modern Logic 2, 113-152. See also Anellis, I. 1992. 'Correction' Modern Logic 3, 98.

Bergmann, M., Mohr, J. and Nelson, J. 1980. The Logic Book, NY: Random House.

Bernays, P. 1936. 'Logical Calculus. Notes by Prof. Bernays with the assistance of F. A. Ficken at Institute for Advanced Study, Princeton, NJ, 1935-36'.

Bohnert, H. 1977. Logic: Its Use and Basis, Washington: University Press of America.

Bonevac, D. 1987. Deduction, Mountain View, CA: Mayfield Press.

Cellucci, C. 1995. 'On Quine's A pproach to Natural Deduction' in P. Leonardi and M. Santambrogio (eds) On Quine: New Essays, Cambridge: Cambridge University Press pp. 314-335.

Chellas, B. 1997. Elementary Formal Logic, Calgary: Perry Lane Press.

Church, A. 1956. Introduction to Mathematical Logic, Vol. I, Princeton: Princeton University Press.

Cooley, J. 1942. A Primer of Formal Logic, NY: Macmillan Co.

Copi, I. 1954. Symbolic Logic, NY: Macmillan Co.

Copi, I. 1956. 'Another Variant of Natural Deduction', Journal of Symbolic Logic 21, 52-55.

Corcoran, J. 1973. 'A Mathematical Model of Aristotle's Syllogistic', Archiv für Geschichte der Philosophie, 191-219.

Curry, H. 1950. A Theory of Formal Deducibility, Notre Dame Mathematical Lectures, No. 6, Notre Dame, IN : Notre Dame University Press.

Curry, H. 1960/61. 'The Inferential Approach to Logical Calculus, Parts I and II', Logique et Analyse 3, 119-136 and 4, 5-22.

Curry, H. 1963. Foundations of Mathematical Logic, NY : McGraw-Hill.

Curry, H. 1965. 'Remarks on Inferential Deduction' in A. Tymieniecka Contributions to Logic and Methodology: In Honor of J. M. Bochenski, Amsterdam: North-Holland.

Feys, R. 1946. 'Les méthodes récentes de déduction naturelle' Revue philosophique de Louvain 44, $370-400$.

Feys, R. 1965. Modal Logics (edited with additions, by J. Dopp) Louvain: E. Nauwelaerts.

Fitch, F. 1952. Symbolic Logic, NY: Roland Press.

Frege, G. 1879. Begriffschrift, Halle: L. Nebert. Translation in Conceptual Notation and Related Articles (translated and edited) T. Bynum, 1972, pp. 101-203, Oxford: Oxford UP.

Gentzen, G. 1934/5. 'Untersuchungen über das logische Schliessen', Mathematische Zeitschrift 39, 176-210, 405-431. Translated as 'Investigations into Logical Deduction' and printed in M. Szabo, The Collected Papers of Gerhard Gentzen, Amsterdam: North-Holland, 1969, pp. 68-131.

Hilbert, D. and Bernays, P. 1934/1939. Gundlagen der Mathematik vol. 1, vol. 2, Berlin;: Springer Verlag. Johnson-Laird, P. N. and Byrne, R. M. J. 1991. Deduction, Hillsdale, NJ : Lawrence Erlbaum.

Jaśkowski, S. 1934. 'On the Rules of Suppositions in Formal Logic' Studia Logica v.1. Reprinted in S. McCall, 1967. Polish Logic 1920-1939, Oxford: Oxford University Press, pp. 232-258.

Kalish, D. 1967. 'Review of I. Copi Symbolic Logic, 2nd Edition'. Journal of Symbolic Logic 32, 252-255.

34 I am grateful to John Corcoran, Renée Elio, Allen Hazen, David Israel, Bernie Linsky, Grigori Mints, Dave Sharp, Patrick Suppes, Geoff Sutcliffe and an anonymous referee for discussions on this topic, and to NSERC grant OPG5525 for its support over the years of my investigations into logic and automated reasoning. This paper was prepared mostly while I was a visitor at Stanford University's Center for the Study of Language and Information. I wish to thank John Perry and Ed Zalta for their hospitality while I visited, and for providing a nice environment in which to pursue intellectual activities of whatever nature. 
Kalish, D. 1974. 'Review of I. Copi Symbolic Logic, 3rd Edition'. Journal of Symbolic Logic 37, 177-178.

Kalish, D. and Montague, R. 1964. Logic: Techniques of Formal Reasoning, NY: Harbourt, Brace, World.

Kearns, J. 1988. The Principles of Deductive Logic, Albany: SUNY Press.

Kleene, S. 1952. Introduction to Metamathematics, NY: van Nostrand.

Klenk, V. 1983. Understanding Symbolic Logic, Englewood Cliffs, NJ : Prentice-Hall.

Kozy, J. 1974. Understanding Natural Deduction, Encino, CA : Dickenson Pub. Co.

Leblanc, H. 1965. 'Minding One's X's and Y's' Logique et Analyse 8, 209-210.

Leblanc, H. and Wisdom, W. 1972. Deductive Logic, Boston: Allyn \& Bacon.

Lemmon, E. J. 1965. Beginning Logic, London: Nelson.

Maslov, S. 1969. 'Relationship between Tactics of the Inverse Method and the Resolution Method' [in Russian] in A. Slisenko (ed.) Zapiski Nauchnykh Seminarov LOMI v.16, pp. 139-146. English translation in Siekmann \& Wrightson 1983. vol. 2, pp. 264-272.

Mates, B. 1965. Elementary Logic, NY: Oxford UP.

Mints, G. 1997. 'Three Faces of Natural Deduction' in Automated Reasoning with Analytic Tableaux and Related Methods: Proceedings of Tableaux 1997, Springer-Verlag Lecture Notes in Artificial Intelligence \#1227. Berlin: Springer-Verlag.

Myro, G., Bedau, M. and Monroe, T. 1987. Rudiments of Logic, Englewood Cliffs: Prentice Hall.

Parry, W. 1965. 'Comments on a Variant Form of Natural Deduction' Journal of Symbolic Logic 30, $119-122$.

Pollock, J. 1969. Introduction to Symbolic Logic, NY: Holt, Rinehart \& Winston.

Prawitz, D. 1965. Natural Deduction: A Proof Theoretical Study, Stockholm: Almqvist \& Wiksell.

Price, R. 1962. 'The Stroke Function and Natural Deduction' Zeitschrift für mathematische Logik und Grundlagen der Mathematik 7, 117-123.

Quine, W. V. 1940. Mathematical Logic, NY: Norton \& Co.

Quine, W. V. 1950a. Method of Logic, NY: Henry Holt \& Co.

Quine, W. V. 1950b. 'On Natural Deduction' Journal of Symbolic Logic 15, 93-102.

Rips, L. J. 1994. The psychology of proof, Cambridge, MA: MIT Press.

Rosser, J. B. 1953. Logic for Mathematicians, NY: McGraw-Hill.

Slater, J. 1966. 'The Required Correction to Copi's Statement of UG' Logique et Analyse 9, 267.

Strawson, P. 1952. Introduction to Logical Theory, London: Methuen.

Suppes, P. 1957. Introduction to Logic, Princeton: Van Nostrand/Reinhold Press.

Tapscott, B. 1976. Elementary Applied Symbolic Logic, Englewood Cliffs: Prentice-Hall.

Thomason, R. 1970. Symbolic Logic, NY: Macmillan.

van Dalen, D. 1980. Logic and Structure, Berlin: Springer-Verlag.

Wilson, J. 1992. Introductory Symbolic Logic, Belmont, CA: Wadsworth. 